\title{
Avaliação do cenário de distribuição de Cirurgiões-Dentistas por especialidades odontológicas no estado da Paraíba, Brasil
}

Evaluation of the scenario of distribution of Dentists by dental specialties in the state of Paraíba, Brazil Evaluación del escenario de distribución de Odontólogos por especialidades odontológicas

en el estado de Paraíba, Brasil

André Rodrigo Justino da SILVA

Rodivan Braz da SILVA ${ }^{2}$

${ }^{1}$ Mestrando do Programa de Pós-Graduação em Odontologia pela Faculdade de Odontologia de Pernambuco, Universidade de Pernambuco (FOP/UPE), 54756-220 Camaragibe - PE, Brasil

${ }^{2}$ Prof.Dr.Adjunto III, Faculdade de Odontologia de Pernambuco, Universidade de Pernambuco (FOP/UPE), 54756-220 Camaragibe - PE, Brasil

\begin{abstract}
Resumo
Introdução: A situação do mercado de trabalho para o Cirurgião-Dentista vem passando por um período de mudanças com aumento da competitividade, predominância do gênero feminino e crescimento do número de especialistas. Objetivo: Caracterizar a distribuição dos cirurgiões-dentistas no estado da Paraíba, no Nordeste brasileiro, de um modo geral, porém com enfoque nas especialidades odontológicas registradas no Conselho Regional de Odontologia. Material e Método: Realizou-se levantamento de dados baseado em pesquisa documental a partir de arquivos públicos com coleta de dados do número de especialistas no estado da Paraíba, com apresentação quantitativa e percentual de cada especialidade de acordo com os sites dos conselhos regional e federal de odontologia. Resultados: Os dados obtidos apontam o total de 5.120 cirurgiões-dentistas registrados no estado da Paraíba, dos quais 1.013 possuem registros como especialistas ativos em 22 especialidades com a predominância do gênero feminino. Assim, a proporção entre profissionais e população a nível estadual é de aproximadamente 1 cirurgião-dentista para cada 735 pessoas. A especialidade com maior registro pelo gênero masculino é a de Cirurgia e Traumatologia, enquanto para o gênero feminino é a Endodontia. As cinco especialidades odontológicas que mais predominam em número geral consistem, em ordem decrescente, em Endodontia, Prótese dentária, Ortodontia, Cirurgia e Traumatologia Bucomaxilofacial e Periodontia. Conclusão: Pode- se concluir que há o predomínio feminino no mercado de trabalho odontológico na Paraíba. Além disso, a especialidade com maior número de profissionais ativos no estado é a Endodontia, sendo também a de maior adesão pelo gênero feminino.
\end{abstract}

Descritores: Recursos Humanos em Odontologia; Especialidades Odontológicas; Mercado de Trabalho.

\section{Abstract}

Introduction: The situation of the job market for the Dentists has been going through a period of changes with increased competitiveness, predominance of the female gender and growth in the number of specialists. Objective: To characterize the distribution of Dentists in the state of Paraíba, in northeastern Brazil, in general, but with a focus on dental specialties registered with the Regional Council of Dentistry. Material and Method: Data collection based on documentary research was carried out from public archives with data collection of the number of specialists in the state of Paraíba, with quantitative and percentage presentation of each specialty according to the websites of the regional and federal councils of dentistry. Results: The data obtained point to a total of 5,120 Dentists registered in the state of Paraíba, of which 1,013 have records as active specialists in 22 specialties with a predominance of females. Thus, the proportion between professionals and population at the state level is approximately 1 dentist for every 735 people. The specialty with the highest record for men is Surgery and Traumatology, while for women it is Endodontics. The five dental specialties that most predominate in general consist, in decreasing order, in Endodontics, Dental Prosthesis, Orthodontics, Oral and Maxillofacial Surgery and Traumatology and Periodontics. Conclusion: It can be concluded that there is a predominance of women in the dental job market in Paraíba. In addition, the specialty with the largest number of active professionals in the state is Endodontics, and it is also the one with the greatest adherence by women. Descriptors: Dental Staff; Specialties, Dental; Job Market.

\section{Resumen}

Introducción: La situación del mercado laboral para el Odontólogo ha pasado por un período de cambios con una mayor competitividad, predominio del género femenino y crecimiento en el número de especialistas. Objetivo: caracterizar la distribución de dentistas en el estado de Paraíba, en el noreste de Brasil, en general, pero con un enfoque en especialidades dentales registradas en el Consejo Regional de Odontología. Material y método: la recopilación de datos basada en la investigación documental se realizó a partir de archivos públicos con la recopilación de datos del número de especialistas en el estado de Paraíba, con presentación cuantitativa y porcentual de cada especialidad de acuerdo con los sitios web de los consejos regionales y federales de odontología Resultados: Los datos obtenidos apuntan a un total de 5,120 Odontólogos registrados en el estado de Paraíba, de los cuales 1,013 tienen registros como especialistas activos en 22 especialidades con predominio de mujeres. Por lo tanto, la proporción entre profesionales y población a nivel estatal es de aproximadamente 1 dentista por cada 735 personas. La especialidad con el registro más alto para hombres es Cirugía y Traumatología, mientras que para mujeres es Endodoncia. Las cinco especialidades dentales que más predominan en general consisten, en orden decreciente, en Endodoncia, Prótesis Dental, Ortodoncia, Cirugía Oral y Maxilofacial y Traumatología y Periodoncia. Conclusión: se puede concluir que hay un predominio de mujeres en el mercado de trabajo dental en Paraíba. Además, la especialidad con el mayor número de profesionales activos en el estado es la Endodoncia, y también es la que tiene una mayor adhesión de las mujeres.

Descriptores: Personal de Odontología; Especialidades Odontológicas; Mercado de Trabajo.

INTRODUÇÃO

A situação do mercado de trabalho para o Cirurgião-Dentista (CD), atualmente vem passando por um período de mudanças, que são decorrentes de situações de ordem econômica e do modelo atual de atenção em saúde bucal. Atrelado à isso está a sucessão de fatos como o aumento da oferta de vagas em cursos de graduação em Odontologia, interiorização das instituições de ensino superior e ampliação dos postos de trabalho no Sistema Único de Saúde (SUS) ${ }^{1,2}$.

A fim de evitar a saturação de 
profissionais, 0 Conselho Federal de Odontologia (CFO) recomendou como parâmetro a ser seguido a relação de $1 \mathrm{CD}$ para cada 2.000 habitantes. Desse modo, o monitoramento do exercício da profissão no país e da densidade de profissionais em nível de estado e município se torna mais fácil para o Conselho ${ }^{3}$.

De modo geral o campo de trabalho em odontologia tem se tornado cada vez mais competitivo, com a predominância do gênero feminino e crescimento do número de especialistas. Por isso, o profissional se vê na obrigação de buscar cada vez mais aperfeiçoarse e capacitar-se para se encaixar as necessidades do mercado. Por outro lado se torna importante que o governo se mostre mais atuante na regulamentação da formação dos $\mathrm{CDs}^{1}$. Sendo assim, a presença dos cirurgiõesdentistas nos serviços e no desenvolvimento de atividades de sua competência, tanto na esfera pública quanto na privada, deve ser cada vez mais analisada e valorizada 4 .

Nessa perspectiva, o objetivo do presente estudo é caracterizar a distribuição dos cirurgiões-dentistas no estado da Paraíba, no Nordeste brasileiro, de um modo geral, porém com enfoque nas especialidades odontológicas registradas no Conselho Regional de Odontologia (CRO). Assim, pretende-se apresentar o cenário quantitativo atual dos profissionais especialistas no estado, pontuando considerações qualitativas acerca dos seus perfis.

\section{MATERIAL E MÉTODO}

O presente trabalho consiste em um estudo de documentação indireta, com levantamento de dados baseado em pesquisa documental a partir de arquivos públicos. A metodologia adotada nesta pesquisa se baseia em metodologias já adotadas em estudos prévios $^{5,6}$, com pequenas alterações. Consiste desse modo na coleta de dados do número de especialistas no estado da Paraíba, com apresentação quantitativa e percentual de cada especialidade de acordo com os sites dos Conselhos Regional e Federal de Odontologia.

Além disso, realizou-se a consulta aos dados referentes ao número de indivíduos que compõem a população do estado através do site do Instituto Brasileiro de Geografia e Estatística $(\mathrm{IBGE})^{7}$, tendo como base o último censo demográfico brasileiro realizado entre agosto e novembro de 2010. Os acessos a todas as plataformas para obtenção dos dados se deram durante o mês de Abril de 2020.

Os resultados obtidos estão apresentados na forma de estatística descritiva, com o destaque para a correlação entre o número de cirurgiões-dentistas e o número de habitantes existentes no estado, além da caracterização levando em conta o aspecto gênero para correlação com os dados encontrados para cada especialidade.

RESULTADOS

Até a data de realização da pesquisa, os dados presentes nos sites do $\mathrm{CFO}^{8}$ e do $\mathrm{CRO} / \mathrm{PB}^{9}$ apontam o total de 5.120 cirurgiõesdentistas registrados, dos quais existem 1.013 registros como especialistas ativos. A predominância de registros de especialistas é do gênero feminino, com $59,24 \%$, enquanto que o gênero masculino possui $40,76 \%$ dos registros ativos (Tabela 1). Os números de CDs especialistas compreendem aproximadamente $20 \%$ do número total de profissionais registrados, portanto atualmente $80 \%$ de inscrições dos cirurgiões-dentistas no conselho são de clínico-gerais.

Tabela 1. Distribuição quantitativa e percentual de CDs especialistas ativos por especialidades odontológicas e divisão quantitativa dos CDs especialistas ativos por gênero e relação percentual entre os CDs especialistas ativos de cada gênero com o total de especialistas ativos de cada especialidade

\begin{tabular}{|c|c|c|c|c|}
\hline $\begin{array}{l}\text { Especialidade } \\
\text { Odontológica }\end{array}$ & $\begin{array}{l}\text { CDs } \\
\text { EA } \\
(\mathbf{n})^{*}\end{array}$ & $\begin{array}{l}\text { CDs } \\
\text { EA } \\
(\%)\end{array}$ & $\begin{array}{l}\text { CDs EA } \\
\text { SM } \\
(\mathbf{n} / \%)^{* *}\end{array}$ & $\begin{array}{l}\text { CDs EA } \\
\text { SF } \\
(\mathbf{n} / \%)^{* *}\end{array}$ \\
\hline Acupuntura & 1 & $0,09 \%$ & $1 / 100 \%$ & $0 / 0 \%$ \\
\hline $\begin{array}{c}\text { Cirurgia e } \\
\text { Traumatologia } \\
\text { Bucomaxilofacial }\end{array}$ & 99 & $9,77 \%$ & $87 / 87,87 \%$ & $12 / 12,12 \%$ \\
\hline Dentística & 19 & $1,87 \%$ & $7 / 36,84 \%$ & $12 / 63,15 \%$ \\
\hline $\begin{array}{c}\text { Dentística } \\
\text { Restauradora }\end{array}$ & 50 & $4,93 \%$ & $9 / 18 \%$ & $41 / 82 \%$ \\
\hline $\begin{array}{c}\text { Disfunção } \\
\text { Temporomandibular } \\
\text { e Dor orofacial }\end{array}$ & 16 & $1,57 \%$ & $2 / 12,5 \%$ & $14 / 87,5 \%$ \\
\hline Endodontia & 133 & $13,12 \%$ & $39 / 29,32 \%$ & $94 / 70,67 \%$ \\
\hline Estomatologia & 20 & $1,97 \%$ & $9 / 45 \%$ & $11 / 55 \%$ \\
\hline $\begin{array}{l}\text { Harmonização } \\
\text { Orofacial }\end{array}$ & o & $0 \%$ & $0 / 0 \%$ & o/ o\% \\
\hline Homeopatia & 0 & $0 \%$ & $0 / 0 \%$ & $0 / 0 \%$ \\
\hline Implantodontia & 86 & $8,48 \%$ & $70 / 81,39 \%$ & $16 / 18,60 \%$ \\
\hline Odontogeriatria & 3 & $0,29 \%$ & $2 / 66,66 \%$ & $1 / 33,33 \%$ \\
\hline $\begin{array}{c}\text { Odontologia do } \\
\text { esporte }\end{array}$ & o & $0 \%$ & $0 / 0 \%$ & $\mathrm{o} / \mathrm{0} \%$ \\
\hline $\begin{array}{l}\text { Odontologia do } \\
\text { trabalho }\end{array}$ & 17 & $1,67 \%$ & $2 / 11,76 \%$ & $15 / 88,23 \%$ \\
\hline Odontologia legal & 15 & $1,48 \%$ & $3 / 20 \%$ & $12 / 80 \%$ \\
\hline $\begin{array}{l}\text { Odontologia para } \\
\text { Pacientes com } \\
\text { Necessidades } \\
\text { Especiais }\end{array}$ & 10 & $0,98 \%$ & $0 / 0 \%$ & $10 / 100 \%$ \\
\hline Odontopediatria & 77 & $7,60 \%$ & $7 / 9,09 \%$ & $70 / 90,90 \%$ \\
\hline Ortodontia & 107 & $10,56 \%$ & $28 / 26,16 \%$ & $79 / 73,83 \%$ \\
\hline $\begin{array}{c}\text { Ortodontia e } \\
\text { Ortopedia Funcional } \\
\text { dos Maxilares }\end{array}$ & 50 & $4,93 \%$ & $14 / 28 \%$ & $36 / 72 \%$ \\
\hline $\begin{array}{l}\text { Ortopedia Funcional } \\
\text { dos Maxilares }\end{array}$ & 6 & $0,59 \%$ & $1 / 16,66 \%$ & $5 / 83,33 \%$ \\
\hline $\begin{array}{c}\text { Patologia Oral e } \\
\text { Maxilo Facial }\end{array}$ & 7 & $0,69 \%$ & $2 / 28,57 \%$ & $5 / 71,42 \%$ \\
\hline Periodontia & 89 & $8,78 \%$ & $36 / 40,44 \%$ & $53 / 59,55 \%$ \\
\hline $\begin{array}{c}\text { Prótese } \\
\text { Bucomaxilofacial }\end{array}$ & o & $0 \%$ & $0 / 0 \%$ & $0 / 0 \%$ \\
\hline Prótese dentária & 120 & $11,84 \%$ & $63 / 52,5 \%$ & $57 / 47,5 \%$ \\
\hline Radiologia & 37 & $3,65 \%$ & $16 / 43,24 \%$ & $21 / 56,75 \%$ \\
\hline $\begin{array}{l}\text { Radiologia } \\
\text { Odontológica e } \\
\text { Imaginologia }\end{array}$ & 24 & $2,36 \%$ & $8 / 33,33 \%$ & $16 / 66,66 \%$ \\
\hline Saúde Coletiva & 27 & $2,66 \%$ & $7 / 25,92 \%$ & $20 / 74,07 \%$ \\
\hline Total & 1.013 & $100 \%$ & $413 / 40,76 \%$ & $600 / 59,24 \%$ \\
\hline
\end{tabular}

Com base nesses dados, das 26 especialidades reconhecidas pelo Conselho 
Federal de Odontologia $(\mathrm{CFO})^{8}$, somente 22 apresentam profissionais registrados para exercício no estado da Paraíba, desse modo, até o momento da pesquisa as áreas de harmonização orofacial, homeopatia, odontologia do esporte e prótese bucomaxilofacial não possuem representação no estado.

Além disso, as cinco especialidades odontológicas que mais predominam em número geral de registrados consistem, em ordem decrescente, em Endodontia, Prótese dentária, Ortodontia, Cirurgia e Traumatologia Bucomaxilofacial e Periodontia.

Por outro lado, levando em conta o fator gênero (Tabela 2), em relação ao número total de CDs especialistas registrados $(n=1.013)$ a especialidade com maior registro pelo gênero masculino é a de Cirurgia e Traumatologia Bucomaxilofacial com $8,58 \%$ dos especialistas ativos. Já para o gênero feminino a Endodontia se mostra como a especialidade de maior registro, com $9,27 \%$.

Tabela 2. Relação percentual CDs especialistas ativos de cada gênero/número total de registros de especialistas ativos $(n=1.013)$

\begin{tabular}{|c|c|c|}
\hline $\begin{array}{l}\text { Especialidade } \\
\text { Odontológica }\end{array}$ & $\begin{array}{l}\text { CDs EASM } \\
\left(^{*}\right)^{*}\end{array}$ & $\begin{array}{c}\text { CDs EASF } \\
\text { (\%)* }^{*}\end{array}$ \\
\hline Acupuntura & $0,09 \%$ & $0,0 \%$ \\
\hline $\begin{array}{c}\text { Cirurgia e Traumatologia } \\
\text { Bucomaxilofacial }\end{array}$ & $8,58 \%$ & $1,18 \%$ \\
\hline Dentística & $0,69 \%$ & $1,18 \%$ \\
\hline Dentística Restauradora & $0,88 \%$ & $4,04 \%$ \\
\hline $\begin{array}{c}\text { Disfunção } \\
\begin{array}{c}\text { Temporomandibular e Dor } \\
\text { orofacial }\end{array} \\
\end{array}$ & $0,19 \%$ & $1,38 \%$ \\
\hline Endodontia & $3,84 \%$ & $9,27 \%$ \\
\hline Estomatologia & $0,88 \%$ & $1,08 \%$ \\
\hline Harmonização Orofacial & $0,0 \%$ & $0,0 \%$ \\
\hline Homeopatia & $0,0 \%$ & $0,0 \%$ \\
\hline Implantodontia & $6,91 \%$ & $1,57 \%$ \\
\hline Odontogeriatria & $0,19 \%$ & $0,09 \%$ \\
\hline Odontologia do esporte & $0,0 \%$ & $0,0 \%$ \\
\hline Odontologia do trabalho & $0,19 \%$ & $1,48 \%$ \\
\hline Odontologia legal & $0,29 \%$ & $1,18 \%$ \\
\hline $\begin{array}{c}\text { Odontologia para Pacientes } \\
\text { com Necessidades } \\
\text { Especiais }\end{array}$ & $0,0 \%$ & $0,98 \%$ \\
\hline Odontopediatria & $0,69 \%$ & $6,91 \%$ \\
\hline Ortodontia & $2,76 \%$ & $7,79 \%$ \\
\hline $\begin{array}{c}\text { Ortodontia e Ortopedia } \\
\text { Funcional dos Maxilares }\end{array}$ & $1,38 \%$ & $3,55 \%$ \\
\hline $\begin{array}{l}\text { Ortopedia Funcional dos } \\
\text { Maxilares }\end{array}$ & $0,09 \%$ & $0,49 \%$ \\
\hline $\begin{array}{l}\text { Patologia Oral e Maxilo } \\
\text { Facial }\end{array}$ & $0,19 \%$ & $0,49 \%$ \\
\hline Periodontia & $3,55 \%$ & $5,23 \%$ \\
\hline Prótese Bucomaxilofacial & $0,0 \%$ & $0,0 \%$ \\
\hline Prótese dentária & $6,21 \%$ & $5,62 \%$ \\
\hline Radiologia & $1,57 \%$ & $2,07 \%$ \\
\hline $\begin{array}{l}\text { Radiologia Odontológica e } \\
\text { Imaginologia }\end{array}$ & $0,78 \%$ & $1,57 \%$ \\
\hline Saúde Coletiva & $0,69 \%$ & $1,97 \%$ \\
\hline TOTAL & $40,76 \%$ & $59,24 \%$ \\
\hline
\end{tabular}

Com relação ao histórico, de acordo com o site do conselho federal de odontologia o ano de maior número de registros no $\mathrm{CRO}$ da Paraíba foi o de 2019, com a inscrição de 437 novos profissionais. Além disso, verificou-se que entre os anos de 1960 e 2020, os anos de maiores registros de especialistas nas cinco especialidades odontológicas mais predominantes ocorreram da seguinte forma: para a Endodontia em 2001 (9); para a Prótese Dentária em 2003 e 2005 (7); para a Ortodontia em 2002 (12); para a Cirurgia e Traumatologia Bucomaxilofacial em 2007 e 2008 (8); para a Periodontia em 2004 (10).

DISCUSSÃo

A população do estado da Paraíba consiste em 3.766.528, de acordo com o último levantamento do IBGE no censo demográfico realizado em $2010^{7}$, desse modo a proporção a nível estadual é de aproximadamente 1 cirurgião-dentista para cada 735 pessoas. Esse achado se relaciona com o estudo de San Martin et al. ${ }^{10}$ que afirmam que o mercado de trabalho está saturado, com um quantitativo maior do que o que é preconizado pela Organização Mundial de Saúde (OMS) e CFO, com algumas regiões apresentando número mais do que suficiente de profissionais, assim como foi encontrado no presente estudo para o estado da Paraíba. Como alternativa os autores apontam a necessidade de estratégias de migração destes CDs para regiões de maior necessidade, a fim e melhorar o futuro da odontologia enquanto profissão.

De acordo com o site do CFO8, no Brasil a especialidade com maior predominância do gênero feminino é a Ortodontia (24,6\%), enquanto que para o gênero masculino a predominância é para a Implantodontia $(21,7 \%)$. No quantitativo geral as especialidades com maior número de registros são Ortodontia $(23,2 \%)$, Implantodontia (13,7\%), Endodontia $(13,5 \%)$, Prótese Dentária $(10,1 \%)$ e Periodontia $(8,3 \%)$. É interessante observar que o estado da Paraíba, apesar de não refletir exatamente esses dados não difere muito, já que a Implantodontia é a segunda especialidade mais procurada pelos profissionais homens e para as mulheres a Ortodontia é também a segunda especialidade com maiores registros. Além disso, das cinco especialidades mais registradas no país somente uma se diferencia do que foi encontrado para a Paraíba: a Implantodontia, que ao nível do estado é substituída pela Cirurgia e Traumatologia Bucomaxilofacial.

Ao se realizar comparação entre o número de $\mathrm{CDs}$ inscritos nas diferentes especialidades no Brasil nota-se a mudança do perfil profissional tanto no aumento do número de registros no país quanto nas especialidades com maior número ativo. Nesse aspecto percebe-se que o cenário atual mudou em comparação há pouco mais de uma década, na qual a Ortodontia, Endodontia e Prótese 
Dentária eram as especialidades predominantes, ao passo que no momento vemos a ascensão da Implantodontia como a segunda especialidade com maior adesão dos cirurgiões-dentistas ocupando a posição que há dez anos talvez não se imaginasse que ocuparia $^{11-15}$. Por outro lado, hoje em dia na Paraíba as três especialidades mais registradas na última década ainda continuam no topo do ranking com mudanças somente nas suas posições. O grande número de profissionais leva a uma discrepância na distribuição entre os setores privados e públicos, com uma concentração maior dos CDs no ambiente privado apesar das potencialidades do mercado no setor público. Isso se deve à insuficiência das políticas de formação profissional voltadas para o SUS e das escassas vagas de emprego ${ }^{16}$.

Os dados apresentados levam a uma reflexão sobre as discrepâncias entre o quantitativo de profissionais na odontologia e o quadro de saúde bucal do Brasil que permanece longe do ideal ${ }^{5}$. A Pesquisa Nacional de Saúde Bucal- Projeto SB Brasil $2010^{17}$ mostra o grau de diferenças na situação de saúde bucal entre as regiões do país e leva a pensar: qual a razão da existência de amplos problemas de saúde bucal, e até a falta de atenção a essas questões em algumas regiões, já que se têm 0 quantitativo de profissionais no mercado de trabalho muito além do mínimo considerado essencial? Os resultados deste estudo poderão servir de base para auxílio no planejamento da oferta de serviços odontológicos, bem como peça para o entendimento da relação entre o quantitativo e o qualitativo na esfera da saúde bucal do estado, sendo de grande interesse para gestores, educadores e profissionais.

\section{CONCLUSÃO}

Os dados obtidos mostram que a maioria dos CDs registrados no conselho regional de odontologia da Paraíba são mulheres. A especialidade com maior número de profissionais ativos no estado é a Endodontia, sendo também a de maior adesão pelo gênero feminino, com destaque para a Cirurgia e Traumatologia Bucomaxilofacial que é a especialidade com maior número de homens. Além disso, observa-se que, quantitativamente pode-se alegar uma saturação de profissionais no estado, porém esse número necessita ser redistribuído para que as políticas de atenção à saúde bucal cheguem a toda população paraibana.

\section{REFERÊNCIAS}

1. Costa DS, Rocha MPO. Cirurgião-Dentista e o mercado de trabalho no brasil: uma revisão sistemática da literatura. Id on Line Rev Mult Psic. 2017;11(38):102-14.

2. Mathias MP, Casani E, Sagaz SM, Lucietto DA. Oferta de cirurgião-dentista do Brasil. IMED. 2016;1-11.

3. Emmi DT, Pinto JCP, Araújo MVA, Barroso RFF. Mercado de trabalho para o cirurgiãodentista no Pará: panorama atual e perspectivas. Rev ABENO. 2019;19(3):26-36.

4. Reis WG, Scherer MDA, Carcereri DL. O trabalho do cirurgião-dentista na Atenção Primária à Saúde: entre o prescrito e o real. Saúde Debate. 2015;39(104):56-64.

5. Figueirêdo Júnior EC, Uchôa NC, Pereira JV. Análise e caracterização do panorama da distribuição de Cirurgiões-Dentistas no Brasil. Arch Health Invest. 2019;8(2):63-7.

6. Figueirêdo Júnior EC, Pereira JV. Análise e caracterização do panorama da distribuição de Cirurgiões-Dentistas no Brasil por especialidades odontológicas. Arch Health Invest. 2019;8(9):465-71.

7. IBGE: Instituto Brasileiro de Geografia e Estatística.2010. Disponível em: https:// cidades.ibge.gov.br/brasil/panorama. Acesso em 15 de abril de 2020.

8. CFO: Conselho Federal de Odontologia. [Internet]. Disponível em: http://cfo.org.br website/ Acesso em 15 de abril de 2020.

9. CRO/PB: Conselho Regional de Odontologia da Paraíba. Disponível em: http://www.cropb. com.br/. Acesso em 15 de abril de 2020.

10.San Martin AS, Chisini LA, Martelli S, Sartori LRM, Ramos EC, Demarco FF. Distribuição dos cursos de Odontologia e de cirurgiõesdentistas no Brasil: uma visão do mercado de trabalho. Rev ABENO. 2018;18(1):63-73.

11. Paranhos LR, Ricci ID, Bittar TO, Scanavini MA, Ramos AL. Análise do mercado de trabalho odontológico na região Centro-Oeste do Brasil. ROBRAC. 2009;18(41):48-55.

12. Parahos LR, Ricci ID, Siqueira DF, Scanavini $M A$, Daruge Júnior $E$. Análise do mercado de trabalho odontológico na região Nordeste do Brasil. Rev Odontol UNICID. 2009;1(2):104-18.

13. Paranhos LR, Ricci ID, Almeida Filho RP, Castro R, Scanavini MA. Análise do mercado de trabalho odontológico na região Norte do Brasil. Rev Odonto. 2009;17(34):27-36.

14. Paranhos LR, Ramos AL, Scanavini MA, Ricci ID. Análise do mercado de trabalho odontológico na região Sudeste do Brasil. Rev Assoc Paul Cir Dent. 2009;63(1):57-63.

15. Paranhos LR, Ricci ID, Scanavini MA, Bérzin $F$, Ramos AL. Análise do mercado de trabalho odontológico na região Sul do Brasil. RFO. 2009;14(1):7-13.

16. Cascaes AM, Dotto L, Bomfim RA. Tendências da força de trabalho de cirurgiões-dentistas no Brasil. Epidemiol Serv Saude. 2018;27(1):1-10. 
17. Brasil. Ministério da Saúde. Secretaria de Atenção à Saúde. Secretaria de Vigilância em Saúde. SB Brasil 2010: Pesquisa Nacional de Saúde Bucal: resultados principais. Brasília: Ministério da Saúde; 2012.116 p.

\section{CONFLITO DE INTERESSES}

Os autores declaram não haver conflitos de interesse

AUTOR PARA CORRESPONDÊNCIA

André Rodrigo Justino da Silva

Rua São José, 429, Bairro Santo Antônio.

58701-120 Patos - PB, Brasil

E- mail: a.rodrigo2010@hotmail.com 